\title{
GNU GPL 2.0 and 3.0: obligations to include licence text, and provide source code
}

\author{
Neil Brown ${ }^{a}$ \\ (a) Solicitor, Privacy, Security and Content Standards, \\ Vodafone Group
}

DOI: $\underline{10.5033 / \text { ifosslr.v2i1.31 }}$

\begin{abstract}
An exploration as to whether, under the terms of the GNU GPL 2.0 and 3.0, a distributor of covered code is entitled to (a) provide a copy of the relevant licence in electronic form, or else via a link to an online location; (b) rely on online distribution of source code without the need to offer source code on a physical medium; and (c) rely on a third party's distribution of the corresponding source code.
\end{abstract}

\section{Keywords}

GNU GPL 2.0, GNU GPL 3.0, compliance

\section{Info}

This item is part of the Articles section of IFOSS L. Rev. For more information, please consult the relevant section policies statement. This article has been independently peer-reviewed.

Following on from his presentation at the Free Software Foundation Europe European Legal Network annual conference in Amsterdam, Neil Brown explores three common compliance topics relating to the GNU GPL 2.0 and GNU GPL 3.0, through an analysis of each of the licences, and the environments in which they were created.

These topics are:

- Obligations of a distributor to distribute the text of a licence along with the covered code;

- Whether a distributor is entitled to rely on online provision of source code, without the need to offer source code on a physical medium; and

- Whether a distributor can rely on a third party's distribution of the corresponding source code of a covered work. 


\section{Obligations of a distributor to distribute a copy of the licence text along with the covered code}

Whether through attempts to lower their environmental impact, to improve the out-of-the-box experience, or otherwise, many companies are increasingly keen to minimise the volume of paperwork provided with each of their products. However, what steps can a company take in respect of the text of the licences of the GNU GPL 2.0 and 3.0?

s1, GNU GPL 2.0' provides:

"You may copy and distribute verbatim copies of the Program's source code ... provided that you ... give any other recipients of the Program a copy of this License along with the Program."

s4, GNU GPL $3.0^{2}$ provides:

"You may convey verbatim copies of the Program's source code ... provided that you ... and give all recipients a copy of this License along with the Program."

Although the scope of the obligation to provide the licence text differs slightly, ${ }^{3}$ the obligation on the distributor ${ }^{4}$ is clear; the distributor is obliged to give a copy of the relevant licence along with the Program.

However, there is no requirement for the copy of the licence to be in physical form; the text simply refers to "a copy", and, on this basis, an electronic copy of the relevant licence is sufficient. Indeed, a licence which required otherwise would be highly impractical in an environment in which software is distributed so widely in electronic format - to require a physical copy of the licence text would be to require a letter or a fax to be sent to each person who downloaded covered code. Electronic distribution could take a number of forms, including incorporation within the interface of a device, which is accessible from another device (e.g. via the "About" section of a router, which has a web interface), storing the licence on, and making it accessible via, the product in question (e.g. an electronic file stored on a mobile phone or a media player, which can be opened by the user on the device itself), or by inclusion on optical media supplied with a product.

Whilst neither licence places a restriction on the format of an electronic copy of the licence, it would be good practice to provide the licence in a format which is accessible to the recipient of the product, such that he is able to read the licence without needing to install non-standard software. For example, if a distributor were to include a copy of the licence on a mobile device, it would advisable to ensure that it could be viewed on that mobile device in an out-of-the-box state. Similarly, the use of restricted formats should be avoided; the Free Software Foundation provides plain text, and other unrestricted, copies of the licences which could be used in pursuit of this

1 http://www.gnu.org/licenses/gpl-2.0.html (All URIs in this article were verified on $14^{\text {th }}$ April 2010)

2 http://www.gnu.org/licenses/gpl.html

3 For example, the GNU GPL 2.0 requires that a copy of the licence is provided to "any other recipients", whereas, under the GNU GPL 3.0, the licence text must be provided to "all recipients".

4 The term "distributor" is used throughout this article to refer to both those who distribute code under the GNU GPL 2.0, and those who convey code under the GNU GPL 3.0. 
objective.

Although electronic distribution of licence text is not restricted by the terms of the GNU GPL 2.0 and 3.0, mere provision of a URL to an online copy of the licence is not a permissible alternative to a physical copy. Each of the licences requires that a copy of the licence be provided "along with the Program"; "along" implies concepts of togetherness and accompaniment, neither of which is satisfied by the inclusion of a URL for a website which a recipient must visit to procure the licence in question. The Preamble to each of the licences confirms this approach, providing that, when one is distributing covered code to another, one must "show them these terms so they know their rights"; merely enabling a user to access a copy of the licence on the Internet neither accompanies the covered code with the licence, nor shows that licence to the recipient of the code.

To conclude this section, then, it is clear that the distributor is required to provide a copy of the licence text along with the covered code, and that, whilst an electronic copy of the licence text is permissible, a mere link to an online copy of the licence is unsatisfactory for the purposes of the GNU GPL 2.0 and 3.0.

\section{Is a distributor entitled to rely on online provision of source code without the need to offer the source code on a physical medium?}

This section seeks to address two common questions in respect of obligations pertaining to source code. Firstly, whether making a copy of the corresponding source code ${ }^{5}$ available online is sufficient to meet a distributor's requirements under each of the licences, and, secondly, whether it is permissible for a distributor to have obligations in respect of source code provision performed by a third party.

\section{Making source code available online}

In respect of offline distribution, s6, GNU GPL 3.0 provides that where object code is conveyed in a physical product, it must be either:

- accompanied by source code on a durable physical medium; or

- accompanied by a written offer either:

- to give the source code on a durable physical medium; or

- offering access to the source code from a network server.

In respect of online distribution, s6 further provides that where object code is distributed by offering access from a designated place, the distributor must offer equivalent access to the corresponding source code, or where distributed by peer-to-peer transmission, the distributor must inform other peers of the online location of the source code.

5 Rather than just the source code form of the object code, a distributor may be required to supply installation scripts and the like, to enable utilisation of the source code. 
As such, a distributor of object code licensed under the GNU GPL 3.0 is never obliged to provide a copy of the corresponding source code on a physical medium, although it remains an option for the distributor to do so, in respect of a distribution of object code on a physical form ${ }^{6}$. However, a distributor is always entitled to rely on the distribution of the appropriate source code from a network server, provided that, where distribution of the object code is in a physical product ${ }^{7}$, the distributor accompanies the object code with a qualifying written offer to make such provision.

Section 3 GNU GPL 2.0 provides that covered code can be distributed in object form, provided that the distribution is accompanied with the complete corresponding machine-readable source code on a medium customarily used for software interchange, or with a written offer to give any third party a copy of the corresponding source code on a medium customarily used for software interchange.

In respect of online distribution of object code, the last paragraph of s3 provides that:

"[i]f distribution of executable or object code is made by offering access to copy from a designated place, then offering equivalent access to copy the source code from the same place counts as distribution of the source code."

As such, where object code is offered online, offering "equivalent access" to the corresponding source code satisfies a distributor's obligation to distribute source code, even if the source code is not actually downloaded by the user downloading the object code.

However, in respect of offline distribution of object code, in order for online availability of source code to be permissible under the GNU GPL 2.0, such online availability must fall within the definition of being "give[n] ... on a medium customarily used for software interchange". As such, one must consider whether making source code available for download by a third party is the same as "giving" that source code to the third party, requiring, as it does, an extra act by that third party to secure the source code. Similarly, "medium" infers a physical object, rather than via a protocol for online transmission. Thus, on a literal interpretation of the licence, online distribution of source code alone ${ }^{8}$ is insufficient for offline distribution of object code.

Whilst such a conclusion may seem incongruous in the light of modern day Internet capability, it makes far more sense when one considers the state of Internet access at the time in which the licence was drafted; in 1991, access to the Internet was far less prevalent than it is today, which meant that, were mere online distribution acceptable, in reality, many recipients of the object form of the code would have been unable to obtain the source code. Since the GNU GPL 3.0 permits online distribution of source code relating to object code distributed offline, on the basis that Internet access is now more prevalent, although far from universal, some might argue that the failure to permit online distribution alone under the terms of the GNU GPL 2.0, whilst legally accurate, is anachronistic, and that, as a result, online distribution of source code in respect of object code distributed offline should be considered sufficient. However, whilst there is merit in

6 Where a distributor provides object code via online access, the distributor is not entitled to rely solely on a written offer to supply a copy of source code on a physical medium, per s6, GNU GPL 3.0.

7 per s6(b)(2), GNU GPL 3.0

8 One could make source code available online in addition to making a written offer to supply source code on a physical medium, if one chose to do so. 
this argument, since the wording of the licence remains unchanged, a distributor adopting this approach would, on a strict legal interpretation of the licence, be exposing itself to risk ${ }^{9}$.

In conclusion, whilst the GNU GPL 3.0 permits mere online distribution of source code, under certain conditions, and the GNU GPL 2.0 permits online distribution of source code where object code is distributed online, online availability of source code is insufficient for offline distribution of object code. However, in reality, it is unclear whether enforcement action would be taken against a distributor of GNU GPL 2.0 covered code, which distributed source code solely from an online location, but was otherwise compliant with the licensing terms.

\section{Using a third party to satisfy source code distribution obligations}

On the basis of the preceding section, under some circumstances, a distributor is entitled to place corresponding source code online, for download by interested recipients. However, what of the situation in which the distributor wishes to rely on a third party to make the source code available? For example, the situation in which a distributor seeks to have its supplier publish the corresponding source code, in respect of object code embedded on a device supplied to the distributor.

The intention of each of the GNU GPL 2.0 and 3.0 is to ensure that the source code is available to anyone who receives (whether from the original distributor or otherwise) the object code. In other words, the Freedoms pertaining to the covered code need to be preserved. With this in mind, and with no express preclusion within the licences, the actual distributor of the source code is immaterial, and a distributor of object code can agree with a third party to provide the corresponding source code on its behalf.

In doing so, however, the distributor of the object code does not transfer its liability as against the owner of the copyright in the code, and, potentially, the recipient of the object code; were the third party with whom the distributor has contracted to fail to provide the source code, the distributor would be liable for the breach of its sub-contractor. As such, a distributor would be advised to consider backing off the risk with the third party, on a contractual basis, in addition to conducting appropriate due diligence to ensure, as far as possible, that the third party's hosting will be reliable. The distributor should also perform a test download of the source code being hosted by the third party, to ensure that it is the compliant corresponding source code.

\section{Conclusions}

Whilst a distributor of code licensed under the GNU GPL 2.0 and 3.0 may provide an electronic copy of the relevant licence text along with the covered code, in a manner which befits the product in question, mere reference to an online copy of the licence is insufficient, since it fails to show the licence to the recipient.

9 If it wished to mitigate the potential impact of this risk, key sections of the community, particularly those involved in licence compliance and enforcement, could make public statements that they will not take enforcement action against a distributor which made its source code available online, without a written offer to provide source code on a physical medium, to give a degree of comfort. However, the absence of a central licensing body for Free software might restrict the success of such an approach. 
A distributor of code under the GNU GPL 3.0 is permitted to make the corresponding source code solely available online whether object code distribution is online or offline, but, if wishing to remain within the wording of the licence, a distributor of code under the GNU GPL 2.0 may only make source code available online where object code distribution is online, or else in addition to providing a written offer or else accompany the object code distribution with source code; mere online distribution of source code does not satisfy the wording of the GNU GPL 2.0 in respect of object code distributed offline.

A distributor may procure a third party to satisfy its obligations under either licence, but the distributor remains liable to the copyright owner, and, potentially, a recipient of the code. As such, a distributor seeking to place reliance on a third party should ensure that it is appropriately protected, in the event that a breach by the third party triggers enforcement action against the distributor.

\section{About the author}

Neil Brown works as a solicitor for Vodafone Group. He is particularly interested in the advancement of knowledge within society, and the role played by copyright in this process. He can also juggle three machetes whilst blindfolded, which is more interesting.

\section{Licence and Attribution}

This paper was published in the International Free and Open Source Software Law Review, Volume 2, Issue 1 (June 2010). It originally appeared online at http://www.ifosslr.org.

This article should be cited as follows:

Brown, Neil (2010) 'GNU GPL 2.0 and 3.0: obligations to include license text, and provide source code', IFOSS L. Rev., 2(1), pp 7 - 12

DOI: 10.5033 /ifosslr.v2i1.31

Copyright $\odot 2010$ Neil Brown.

This article is licensed under a Creative Commons UK (England and Wales) 2.0 licence, no derivative works, attribution, $C C-B Y-N D$.

As a special exception, the author expressly permits faithful translations of the entire document into any language, provided that the resulting translation (which may include an attribution to the translator) is shared alike. This paragraph is part of the paper, and must be included when copying or translating the paper.

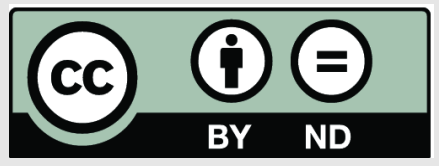

